\title{
Analysis of Observations Acquired from Digieye and Macbeth
}

\author{
a Abdul Malik Rehan Abbasi \\ a: Department of Textile Engineering, Balochistan University of Information Technology, Engineering \\ and Management Sciences, 87300, Quetta, Pakistan
}

\begin{abstract}
The aim of paper was to indicate a compatibility of results obtained from two kinds of equipment: Macbeth and DigiEye. To accomplish this, the selected materials were tested on both devices. DigiEye is a computer controlled digital camera used to measure the colour changes caused by special fabric treatment. The data that can be obtained are the reflectance or colourimetric results. The operating principle of Macbeth spectrophotometer is based on the quantitative measurement of transmission and reflection of the light by the sample. Macbeth has been used to test not only the textile materials but also for other solids and liquids since the middle of the twentieth century. Colour measurement in the CIELab system and comparison of results from both DigiEye and Macbeth indicates the differences or correlation between these two devices. The objective of this work was to analyse the possibility of a wide application of the DigiEye system in textile industry for evaluation of colour and its durability. The study demonstrated the significant differences in the results obtained from both the instruments, however, these differences follow very specific shift of the lightness, chromaticity and hue of the colour and needs further investigation.
\end{abstract}

Keywords- DigiEye, Macbeth, CIELab, spectrophotometer, colorimeter

Date Received 16 May 2019

Date Accepted 13 June 2019

Date Published 5 July 2019

\section{INTRODUCTION}

$\mathrm{T}$ he perception of colour is an individual sensation of the observer. Determination of colour is dependent on the spectral light radiation, the amount of light energy and the presence of other composite colours. The radiation can pass through the transparent objects, be reflected from the opaque surface or partially absorbed. Textile samples are typically measured in the range of wavelength $(\lambda)$ from $400 \mathrm{~nm}$ to 700 $\mathrm{nm}$. This follows from the fact that most of the people can see within the visible range that is between $\lambda=400 \mathrm{~nm}$ and $\lambda=700$ $\mathrm{nm}$ [1]. The colour assessment of textiles is important from the point of view of the user. Consumers pay attention to the product appearance and colour uniformity. Therefore, it is important to have systems that allow for the objective colour evaluation. Modifications of colour schemes allowed creating the trichromatic Red-Blue-Green (RGB) coordinate system, which is the basis of the trichromatic values (XYZ). Currently, the colour measurement adopted the CIELab system introduced by the International Commission on Illumination (Commission Intrenationale de l'Eclairage). CIELab system is based on assigning attributes of colour such as: lightness (L), saturation (chroma), and hue, which are shown in Figure 1 [1].

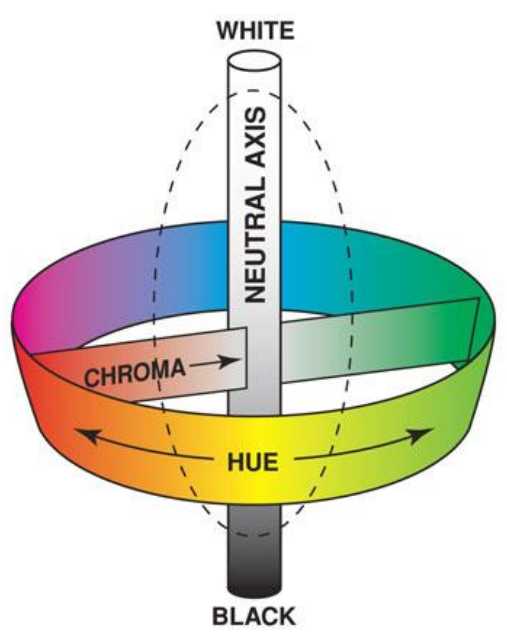

Figure 1: Schematic diagram showing a graphic colour interpretation [2].

During the measurement of colour we should have in mind the type of illuminants. CIE Commission has identified patterns for the white light, called standards illuminants.

The "illuminant A" depicts yellow-orange light of wolfram bulb filled by gas with the colour temperature $2856 \mathrm{~K}$ and also called warm light. The "illuminant C" represents the tungsten light bluish (approximately daylight, dispersed with no direct sun light) with the colour temperature $6774 \mathrm{~K}$ and also called cold light. The "illuminant D" shows averaged daylight from different times of the day based on the actual measurement of spectral light with the colour temperature $6504 \mathrm{~K}$ and also 
written as (illuminant D65) and the "illuminant F" which resembles fluorescent light.

CIELab system make the colour matching much easier. It is very important to map the colour of the pattern during the production process of dyeing. The difference between the colour and pattern of the sample is determined by the following formula:

$$
\Delta E=\sqrt{(\Delta L)^{2}+(\Delta a)^{2}+(\Delta b)^{2}}
$$

$\Delta \mathrm{E}$ - the difference between the colour of the sample and the pattern [3, 4]. CIELab colour coordinates measurements and determination of the colour differences is performed according to PN-EN 105-J01: 2002 and BS-EN105-J03, 2009.

Taking above into consideration, the colour measurement of textile substrates should be taken as one of the most important aspects of quality assessment. Different methods are employed to assess the colour of the textile goods. Among them instrument based assessments are most commonly carried out by spectrophotometers such as Macbeth ${ }^{\circledR}$ and Datacolor ${ }^{\circledR}$ etc.

There are some reports elucidating the application of DigiEye system in measurement of colour of textile materials. One of the advantages of DigiEye is that it can analyse the colour of the larger area of the substrate, which is of principal importance taking into consideration the pattern of the printed fabrics that might have large repeat (Figure 4), exceeding the measurement area of the spectrophotometers (e.g., $30 \mathrm{~mm}$ in diameter).

Thus, the main aim of the work is to measure the colour of the printed fabric by DigiEye and comparing the results with those acquired from Macbeth.

\section{MEASUREMENT PRINCIPLES \\ 2.1 SPECTROPHOTOMETER}

For the measurement of colour changes in the textile industry, devices of spherical geometry is commonly used. Those devices are equipped with a so-called Ulbricht sphere, which diffuses the light. The interior of the sphere is covered by the white layer with parameters similar to the ideal white. The basis for calculation of parameters in the CIELab system is the measurement of reflectance by the spectrophotometer. The idea of a spectrophotometer is the light diffusion inside the sphere. The measurement made by the Macbeth 2020 spectrophotometer is carried out with the geometry $\mathrm{d} / 8^{\circ}$. It means, first spread the light integrating sphere and then the reflected from the sample beam is collected at an angle of $8^{\circ}$. This eliminates the mirror effect of the surface however, the beam reflected by the sample is collected at the angle of $8^{\circ}$ (Figure 2.) [4, 5].

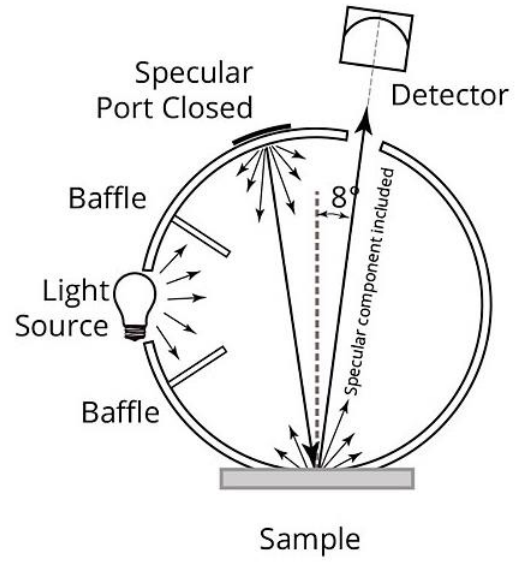

Figure 2. The geometry used for the reflection of light $\mathrm{d} / \mathrm{8}^{\circ}$ [6].

\subsection{DigiEye}

DigiEye uses a new technology of colour measurement, which is based on taking a picture of the tested object. This technique allows for the non-contact measurement in a few seconds. The cabinet is lighted by a combination of fluorescent D65 illuminant and additive LEDs to allow the production of calibrated A-rated D65 simulator. Special colour plate called DigiTizeris is used for the calibration. The results are obtained on the basis of indicating the sample point on the computer screen or selecting of the whole sample area. The DigiEye device can also obtain an image of the surface gloss and texture. The option used for measurement of colour is called the Colour Measurement. The measurement data are reported in terms of colourimetric values. DigiEye calculates also the average colour of all the pixels within the selected area of the image or corrected to take into account surface effects of the substrate [7].

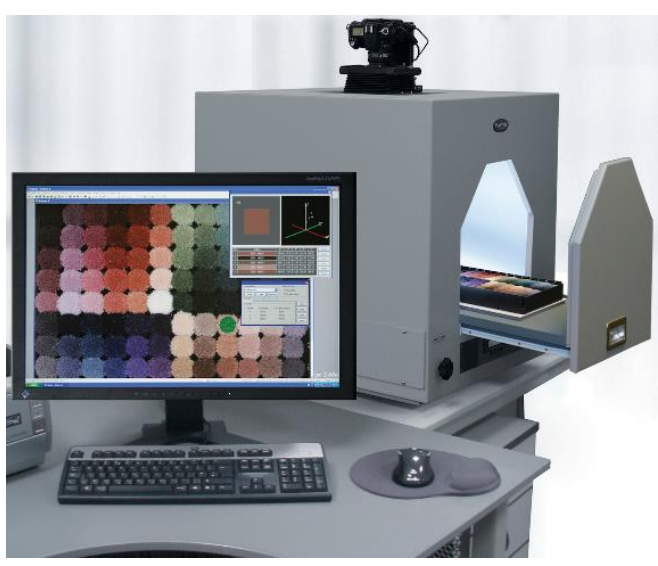

Figure 3. The DigiEye device

\section{OBJECT OF EXAMINATION}

Examination was performed in a spectrophotometer on several composite fabrics, to prevent light passing. This is done in order to eliminate the influence of the substrate. To avoid irregularity 
of dyeing and the difference in the structure of the fibers, the measurement is averaged. Therefore, the test should be repeated six times. Measurements made on DigiEye carried out for the angular positioning of the light source. An analysis of results obtained from the DigiEye and Macbeth was based on examination of 7 fabric samples of the same textile materials. There was also measured the spectrophotometer pattern: GUM 200. Colour assessment was done for fabrics destined for the military products - camouflage and technical textiles - Velcro.

The fabric samples are a blend of cotton and polyamide 6.6 at a ratio of $50 / 50 \%$ with the warp count of $17 \times 2$ tex and weft count 40 tex. The fabric sample was woven in combination of twill and rep weave with 36 ends/cm and 20.7 picks $/ \mathrm{cm}$. The mass of the fabric sample was 220 g. $\mathrm{m}^{-2}$ and thickness was $0.436 \mathrm{~mm}$.

\section{RESULTS AND ANALYSIS}

A primary differences between results obtained from both devices used for tests are based on the lighting the samples. In the spectrophotometer sphere the diffused light is directed at the examined sample. In the DigiEye cabinet there are two especially prepared lamps, so the sample is illuminated from two sides. The different way of the sample illumination causes a completely different reflection; and therefore, the different measurement results. The additional complication could be the texture of fabric in the case of camouflage pattern illustrated in Figure 4.

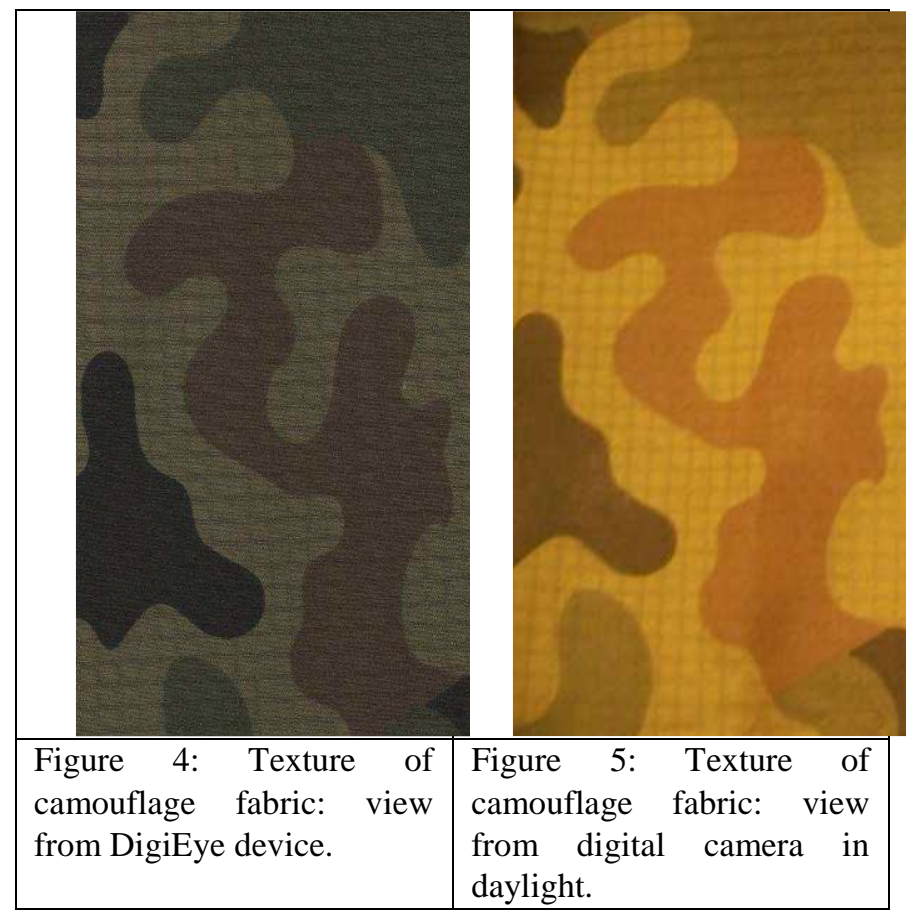

Colour measurement on both devices showed the different colour coordinates. This is probably caused by a difference in the method of sample illumination. It led us to the implementation of tests to determine differences between the two samples. The comparison of colour coordinates was performed on both devices for all the samples; whereas in this case, there measured difference $\Delta \mathrm{E}$ between only two fabrics.
The results from both instruments are shown in Table 2.

In the second method, the differences obtained from the two devices are almost identical. This may provide a way to use the DigiEye device for measuring colour changes. The differences in the measurements of the colour coordinates of the fabric sample is in fact due to the certain differences in the shape of the reflectance curves between the DigiEye and spectrophotometer. However, a strong correlation was found between the coordinates acquired from both instruments regarding lightness and chromaticity coordinates. As correlation coefficients between the various colour parameters are not of primary importance in colour evaluation, a more sophisticated methodology can be employed to assess the degree of agreement between DigiEye and Macbeth in terms of colour difference calculation.

\section{CONCLUSIONS}

The spectrophotometer (in this study Macbeth) are widely used for fabric colour measurements, however, it has some limitations that can be excluded by application of comparatively different colour measurement system that is DigiEye. Although this device is commonly used for the evaluation of the difference in products from textile substrates, such as foodstuffs etc. but efforts have been made to use it for the assessment of textile substrates.

The applied research methodology has shown that there are differences in the absolute values of sample colour coordinates for both equipment. This is due to the different ways of sample illumination in both methods. Spectrophotometer acting principle bases on the spectral characteristics, which is the base to calculate XYZ colour coordinates; and next, to convert them into the CIELab system. It can be concluded that the colour measurement on device DigiEye device is done by a comparison of the sample in relation to the colour plates DigiTyzer. Measuring the differences in colour between two different fabrics on both devices allows establishing the similar values.

\section{ACKNOWLEDGEMENT}

The work is conducted at the Department of Material Engineering, Technical University of Liberec, Czech Republic. 
Table 1: The comparison results of tested fabrics

\begin{tabular}{|l|c|c|c|c|c|c|c|}
\hline Sample symbol & \multicolumn{3}{|c|}{ Macbeth } & \multicolumn{3}{c|}{ DigiEye } & $\begin{array}{c}\text { Difference } \\
\text { between } \\
\text { devices }\end{array}$ \\
\hline & L & a & b & L & a & b & $\Delta E$ \\
\hline $\begin{array}{l}\text { Camouflage pattern light } \\
\text { green }\end{array}$ & 35.89 & 1.46 & 11.20 & 32.87 & 0.57 & 14.33 & 4.4 \\
\hline $\begin{array}{l}\text { Camouflage pattern dark } \\
\text { green }\end{array}$ & 29.36 & -1.17 & 7.66 & 25.999 & -2.49 & 13.23 & 6.6 \\
\hline Camouflage pattern brown & 29.47 & 4.55 & 7.86 & 26.51 & 4.33 & 12.62 & 5.6 \\
\hline Camouflage pattern black & 22.08 & 0.16 & 2.54 & 18.32 & -2.67 & 4.11 & 5.0 \\
\hline Pattern GUM 200 & 70.95 & -34.28 & -14.94 & 74.46 & -37.70 & -13.19 & 3.8 \\
\hline Navy blue & 16.21 & 0.66 & -1.21 & 17.98 & -0.98 & -2.68 & 2.8 \\
\hline Velcro loop & 59.62 & 2.86 & 13.56 & 61.84 & 4.82 & 13.21 & 3.0 \\
\hline Velcro hook & 58.75 & 3.11 & 13.36 & 60.96 & 4.75 & 13.12 & 2.8 \\
\hline
\end{tabular}

Table 2: Comparison of $\Delta \mathrm{E}$ parameter

\begin{tabular}{|c|c|c|c|c|c|c|c|}
\hline \multirow{2}{*}{ Device name } & \multicolumn{3}{|c|}{ Black fabric } & \multicolumn{3}{c|}{ Navy blue } & $\begin{array}{c}\text { Difference } \\
\text { between } \\
\text { fabrics }\end{array}$ \\
\hline & L & a & b & L & a & b & $\Delta \mathbf{E}$ \\
\hline Macbeth & 15.60 & 0.72 & -1.12 & 19.01 & 0.43 & -3.09 & 3.9 \\
\hline DigiEye & 15.530 & 1.600 & -0.210 & 18.280 & 1.890 & -2.350 & 3.5 \\
\hline
\end{tabular}

\section{References}

[1] X. Zhang and B. A. Wandell, "A spatial extension of CIELAB for digital color-image reproduction,"

Journal of the Society for Information Display, vol. 5, pp. 61-63, 1997.

[2] B. J. Thomas, T. W. Graham, and D. C. Briggs, "Light source having desired color temperature and chromaticity," ed: Google Patents, 1992.

[3] A. R. Robertson, "The CIE 1976 Color-Difference Formulae," Color Research \& Application, vol. 2, pp. 7-11, 1977.

[4] R. Berns, F. Billmeyer, and M. Saltzman, "Measuring color," Billmeyer and Saltzman principles of color technology. New York: John Wiley, pp. 75-105, 2000.

[5] S. G. Kandi, "The effect of spectrophotometer geometry on the measured colors for textile samples with different textures," J. Eng. Fibers Fabr, vol. 6, pp. 70-78, 2011.

[6] P. Ehbets, "Spectrophotometer and its use," ed: Google Patents, 2005.

[7] M. Matusiak, "DigiEye Application In Cotton Colour Measurement," Autex Research Journal, vol. 15, pp. 77-86, 2015. 